\title{
La médecine d'urgence au Canada : un appel aux armes
}

\author{
James Ducharme, MD, CM ${ }^{*}$; Riyad B. Abu-Laban, MD, MHSc ${ }^{\dagger}$; Marco L.A. Sivilotti, MD, MSc ${ }^{\dagger}$; \\ Brian H. Rowe, MD, $\mathrm{MSc}^{\dagger}$
}

\section{English version on page 8}

E $\mathrm{n}$ tant que rédacteurs principaux du Journal canadien de la médecine d'urgence (JCMU), nous avons remarqué, au cours des 2 dernières années, des tendances inquiétantes relatives aux bénévoles sur lesquelles le journal compte tant. Les pairs examinateurs déclinent les demandes d'évaluation des manuscrits à un rythme alarmant, ce qui force notre équipe éditoriale à contacter plus de 10 personnes pour obtenir les 2 ou 3 évaluations requises. Les rédacteurs responsables des décisions sont également moins disponibles et plus lents à effectuer leurs tâches. Qui plus est, il s'avère difficile, voire impossible, de trouver des rédacteurs associés pour prendre en charge de nouvelles initiatives ou pourvoir à des postes vacants de plus en plus nombreux. En outre, on observe une réponse tiède aux demandes de communications pour de nouvelles sections, comme la nouvelle rubrique de la page de quatrième couverture, Virage radical. Maintenant que le JCMU est indexé et reconnu internationalement comme revue scientifique de médecine d'urgence, ces bénévoles ont-ils tous perdu leur sens de l'urgence ou le désir de contribuer? $\mathrm{Ou}$ s'agirait-il de problèmes émanant de questions plus larges et plus fondamentales touchant non seulement le volet enseignement de la médecine d'urgence, mais aussi la médecine d'urgence en général au Canada?

Pour comprendre notre situation actuelle, il faut réfléchir sur notre évolution. Contrairement à la médecine d'urgence aux États-Unis, les soins cliniques et l'enseignement médical constituaient les piliers fondateurs de cette discipline au Canada. L'Association canadienne des médecins d'urgence (ACMU) a lancé sa première « tournée de formation » dans les années 80 et elle continue de consacrer beaucoup d'énergie et de ressources au développement professionnel continu (DPC).
La recherche est vite devenue le troisième pilier, grâce à des initiatives telles que le volet recherche au congrès scientifique annuel de l'ACMU, la création du JCMU et un concours annuel de subventions de recherche. La communauté de médecine d'urgence au Canada peut se vanter d'avoir des individus de renommée internationale dans tous ces domaines.

D'une part, l'ACMU doit, de toute évidence, continuer de favoriser le DPC auprès de ses membres. D'autre part, maintenant que la presque totalité des médecins d'urgence qui amorcent leur carrière sont formés en résidence et obtiennent leur certificat des 2 voies de certification, on dispose de solides arguments pour que le DPC n'occupe plus une place centrale à l'ACMU. Cette association et la communauté de la médecine d'urgence en général devraient plutôt mettre davantage l'accent sur le quatrième pilier, à savoir l'arène politique et administrative. Notre manque collectif d'expansion dans ce domaine demeure un obstacle considérable à l'avancement de la médecine d'urgence au Canada. Bien qu'au départ l'American College of Emergency Physicians ait été en retard sur le Canada en matière de recherche, il est, depuis ses débuts, au premier rang en ce qui concerne ce quatrième pilier. C'est probablement la principale raison pour laquelle la médecine d'urgence connaît un tel essor au sud de notre frontière. Contrairement aux États-Unis, où existent plusieurs organisations nationales de médecine d'urgence pour faire progresser et développer différents aspects de cette discipline, le Canada n'en a qu'une. Cette situation n'est pas susceptible de changer dans un proche avenir. Par conséquent, l'ACMU se doit d'avoir un mandat clair et de bénéficier d'une participation active des médecins pour l'ensemble de ces domaines. Malheureusement, ce

\footnotetext{
*Rédacteur en chef, JCMU, †Rédacteurs associés principaux, JCMU
}

Les opinions exprimées dans les éditoriaux sont celles des auteurs; elles ne reflètent pas nécessairement celles du JCMU ou de l'Association canadienne des médecins d'urgence. 
n'est qu'une minorité des médecins travaillant dans les services d'urgence du Canada qui sont membres de l'ACMU et ils sont encore moins nombreux à posséder une expertise relative à ce quatrième pilier.

Il existe certes des exceptions notables, mais de nombreux médecins d'urgence du milieu universitaire au Canada hésitent à participer à des activités politiques et administratives liées à l'avancement de la médecine d'urgence. Au cours des 20 dernières années, seuls quelques présidents de l'ACMU provenaient de centres hospitaliers universitaires et des membres du Conseil qui étaient actifs sur le plan de l'enseignement étaient aussi rares, malgré les efforts de l'ACMU pour changer ce constat. Comme la plupart des grands hôpitaux au Canada sont aussi des centres d'enseignement, il est essentiel d'assurer une représentation politique et administrative de la médecine d'urgence dans ces établissements. Or, même parmi les groupes urbains de médecins d'urgence à temps plein, le taux d'adhésion à l'ACMU est loin d'être optimal. Alors, quelle organisation autre que l'ACMU nous représentera? Si l'ACMU ne peut compter sur une base solide de membres actifs, et la voix et les ressources financières qui en découlent, elle continuera de se démener pour avoir la volonté politique et la force de faire avancer nos principaux chevaux de bataille. Comme nous l'avons vécu auparavant, le résultat est que les autres - des groupes mieux organisés - dicteront notre direction. Bien que l'ACMU soit fière d'être une société nationale de spécialité, il semble que les médecins d'urgence du milieu universitaire croient de plus en plus qu'elle est sans importance ou qu'elle survivra sans eux. Est-ce possible que ce journal soit aussi victime de cette apathie mal placée?

Il est difficile de considérer les défis de la médecine d'urgence en milieu universitaire sans tenir compte de notre situation clinique. Depuis la création de notre discipline, on croyait que personne ne pourrait pratiquer la médecine d'urgence pendant toute sa carrière sans être victime d'un « épuisement professionnel ». La recherche et l'expérience ont pourtant montré que c'était faux. Récemment, toutefois, on entend parler de nombreux médecins d'urgence qui planifieraient leur sortie. Au cours des dix dernières années, les conditions de travail se sont considérablement détériorées en raison de l'engorgement des urgences, des temps d'attente et des pénuries de personnel. En outre, l'étendue du champ d'exercice des médecins d'urgence change constamment, ce qui crée une rupture entre ce que l'on enseigne aux résidents et ce qu'ils vivent réellement quand ils commencent à pratiquer la médecine d'urgence. On imaginait que la médecine d'urgence serait le filet de sécurité pour les patients gravement malades ou blessés, mais elle devient de plus en plus le filet de sécurité pour l'ensemble du système de santé. Qui en fait définit ce qu'est la médecine d'urgence? Il semble que ce soit tout le monde sauf nous.

Nos enjeux cliniques ont été bien documentés et étudiés, mais ils n'ont pas encore fait l'objet de corrections significatives et ils continuent de se détériorer. Cela met en évidence notre manque de progrès sur le plan politique, en particulier notre incapacité à mettre en application des normes significatives contre lesquelles les soins d'urgence peuvent être mesurés. Il est sans doute vrai de dire que l'ACMU a manqué à cet égard. Mais qui forme l'ACMU et qui est responsable de corriger ce manque? Peut-être plus important encore, qui ne fait pas partie de l'ACMU? Si l'on pointe le miroir dans l'autre direction, il faut aussi se demander si l'ACMU est aussi transparente qu'elle devrait l'être sur la portée de son mandat et les personnes qu'elle représente. Il est frappant de constater que ni sa vision ni son énoncé de mission (disponible à www.caep.ca) ne font référence à la médecine d'urgence en milieu universitaire, au processus de certification ou à la médecine d'urgence comme spécialité.

La structure de nos deux programmes de formation indépendants constitue peut-être un obstacle supplémentaire au développement de la force politique et administrative de la médecine d'urgence au Canada. En théorie, le programme CCMF (MU) du Collège des médecins de famille du Canada vise à produire des médecins praticiens alors que le programme du Collège royal des médecins et chirurgiens du Canada (CRMCC) a été conçu pour produire des consultants et des chefs de file en médecine d'urgence. Le volet d'un an en médecine d'urgence du programme CCMF (MU) ne permet pratiquement pas d'acquérir des compétences non cliniques. Naturellement, les membres du corps professoral encouragent les résidents du programme du CRMCC à développer un créneau ou des compétences hors du domaine clinique. Malheureusement, presque aucun résident ne s'en sert pour accroître sa compréhension du système de santé ou apprendre comment travailler avec succès dans le système afin d'améliorer la médecine d'urgence. D'une part, on note un surplus de formation en recherche, en enseignement et en compétences techniques; d'autre part, la formation en gestion des affaires, en gestion des changements et en formation relative aux opérations est rare. Il se peut que collectivement nous ayons surspécialisé la médecine d'urgence 
trop tôt, avant d'avoir établi la masse critique de spécialistes en médecine d'urgence nécessaire au Canada. En outre, le développement d'un créneau peut offrir la possibilité de pratiquer une spécialité en autonomie, parfois dans de meilleures conditions de travail et selon une meilleure rémunération. Il ne faut donc pas se surprendre que l'on perde de plus en plus de précieux récents diplômés du CRMCC en médecine d'urgence, surtout quand les conditions dans nos urgences sont intenables.

Sur le plan politique, nombreux sont ceux qui pensent que nous sommes incapables d'influencer la réforme des soins de santé. Les succès que notre spécialité a connus (contrôle des armes à feu, accès aux tests diagnostics et législation sur le port du casque) réfutent ce mythe. Malgré cela, certains de nos plus brillants esprits évitent délibérément tout ce qui touche à la réforme des soins de santé. Pourquoi? On compte parmi nous d'excellents administrateurs, mais il semble que peu sentent qu'ils sont faits pour ce domaine. Certes, peu ont reçu une formation officielle pour assurer leur succès et une longue carrière. Les résidents observent une absence de modèles dans ce domaine. Il n'est donc pas surprenant qu'ils entrent rarement dans cette arène. Si nous ne descendons pas dans l'arène politique et ne réclamons pas notre place, qui va parler en notre nom et en celui de nos patients? Qui va entreprendre les étapes nécessaires à la progression de la médecine d'urgence vers la discipline qu'elle devrait être?

Avec d'un côté un manque de leaders politiques et de l'autre de piètres conditions de travail, il n'est pas étonnant que le milieu universitaire manifeste son mécontentement. En outre, il n'existe pas de modèle uniforme pour le financement non clinique. De nombreux groupes universitaires n'ont pas encore le statut de département et, par conséquent, ont moins d'influence politique dans leur milieu. À l'heure actuelle, nous n'avons pas de chaires fondées en médecine d'urgence, nous avons une chaire de recherche du Canada, une poignée de chercheurs de carrière et quelques chaires départementales bénéficiant d'un appui financier. De nombreux groupes, y compris ceux de centres hospitaliers universitaires, sont en difficulté. Des changements pour ces groupes ne pourront être enclenchés qu'avec l'aide d'une masse critique de médecins d'urgence du milieu universitaire disposant d'un financement sûr et de chefs de file qui sont des experts en administration de la santé. Nous avons besoin de visionnaires qui peuvent faire de leur vision une réalité, de personnes qui travaillent au niveau national, au-delà de leur propre groupe.

Si l'on veut que l'ACMU soit le gouvernail dont nous croyons que notre navire collectif a besoin, elle doit être investie d'un mandat plus clair, d'un mandat qui inclut la médecine d'urgence en milieu universitaire. Mais pour ce faire, il faut que les médecins d'urgence du milieu universitaire s'engagent plus à fond. Cela présente un dilemme. Il faut que des personnes ayant une profonde conviction à l'égard de la médecine d'urgence nous guident à travers ces moments difficiles. Ce n'est pas le moment de battre en retraite. Cela exige que les médecins continuent de pratiquer la médecine d'urgence et s'engagent à apporter les changements nécessaires plutôt que de partir parce que cela n'est plus aussi amusant qu'avant. Ces personnes et leurs succès redynamiseront les autres. Nous verrons tous que nous pouvons apporter notre contribution et que nos efforts ne sont pas vains. Maintenant que nous avons acquis une certaine maturité et de l'expérience en tant que profession, ce n'est pas le temps d'abandonner la partie. C'est le temps de mettre à profit notre expérience pour faire des changements novateurs qui amélioreront nos conditions de travail, les programmes universitaires et les soins aux patients.

D'où cet appel aux armes. Nous constatons une apathie grandissante, une participation chancelante, et de nombreux médecins qui se diversifient et se concentrent sur autres choses que l'amélioration de la médecine d'urgence au Canada. Il est temps pour nous tous de reconnaître qu'il faut renforcer le pilier politique et administratif de la médecine d'urgence. Prenons donc tous le temps d'envisager comment nous pouvons apporter notre aide. Le succès de nos deux institutions canadiennes, l'ACMU et le JCMU, est d'une importance capitale pour nous tous, indépendamment du lieu ou de la nature de notre travail. Pour être de véritables défenseurs de la médecine d'urgence et des droits de nos patients, nous devons faire davantage sur le plan collectif. Qui sait? Peut-être qu'alors nous n'aurons plus à nous soucier de l'enthousiasme et des délais d'exécution des pairs examinateurs et des rédacteurs du $7 C M U$...

\section{Conflits d'intérêts : aucun déclaré.}

Mots-clés : médecine d'urgence, administration, représentation, milieu universitaire

Correspondance : $\mathrm{D}^{\mathrm{r}}$ James Ducharme; cjem@telus.net 